\title{
Ascenso y deClive de Estados Unidos EN LA HEGEMONÍA MUNDIAL
}

\author{
Alejandro Dabat ${ }^{\mathrm{a}}$ y Paulo Leal ${ }^{\mathrm{b}}$
}

Fecha de recepción: 11 de noviembre de 2018. Fecha de aceptación: 6 de junio de 2019.

http://dx.doi.org/10.22201/iiec.20078951e.2019.199.67934

Resumen. El presente artículo integra un comparativo de dos épocas distintas en el orden económico mundial: la crisis de hegemonía inglesa de fines del siglo xIx junto con la consolidación de la hegemonía estadounidense al final de la Segunda Guerra Mundial, y el proceso actual de crisis de la hegemonía estadounidense a partir de 2001 a la fecha. El planteamiento parte de una visión estructural de la economía en la que el sistema productivo mundial se transforma a lo largo de distintas etapas históricas y junto con otros elementos como el orden monetario mundial y el liderazgo político, institucional y militar, transforma el orden económico y la hegemonía. Aplicando esa metodología se concluye que ambos periodos muestran diversas similitudes, pero también profundas diferencias, y que en la actualidad el orden mundial se encuentra en un proceso de cambio hacia la multipolaridad.

Palabras clave: crisis de hegemonía; economía mundial; multipolaridad.

Clasificación JEL: F02; O51; P51; P52.

\section{THE RISE AND FALL OF THE UNited STATES IN GLOBAL HEGEMONY}

\begin{abstract}
The present article brings together a comparison of two distinct epochs in the world economic order: the crisis of English hegemony at the end of the $19^{\text {th }}$ century, along with the consolidation of American hegemony at the end of World War II and the contemporary process of the crisis of American hegemony beginning in 2001 and continuing today. This approach is based on a structural perspective of the economy in which the global productive system is transformed across the span of different historical periods, and which along with other elements - such as the global monetary order and political, institutional and military leadership- transforms the economic order and hegemony. Applying this methodology leads to the conclusion that the two periods show various similarities, though there are also profound differences, and that currently the global order is in the process of changing towards multipolarity.

Key Words: crisis of hegemony; world economy; multipolarity.

${ }^{a}$ Universidad Nacional Autónoma de México (UNAM), Instituto de Investigaciones Económicas, México; ${ }^{b}$ unam, Facultad de Economía, México. Correos electrónicos: adabatlat@gmail.com y phleal77@gmail.com, respectivamente. Con apoyo de los Proyectos PAPIIT (DGPA), IN304019 "El siglo xx en perspectiva actual" y PAPIME PE304919 "Análisis de la estructura económica en el proceso de integración de la economía mexicana a la estadounidense (1982-2017)”. Este trabajo fue realizado con la colaboración de Enrique Arriola y Luis Romero.
\end{abstract}




\section{INTRODUCCIÓN}

Este artículo tiene como objetivo interpretar el auge y la decadencia de Estados Unidos de América (EUA) como potencia hegemónica mundial. En un principio se plantea que el proceso de ascenso de la nación americana se debió a la decadencia de la hegemonía inglesa y, aunque su decadencia está por igual acompañada del auge de China, ambos procesos presentan profundas diferencias; se observa muy complicado que de una forma mecanica la decadencia de EUA vaya a dar paso a una hegemonía china en el corto plazo, pero sí que la hegemonía absoluta de EUA ha desaparecido por un proceso de declinación propio y por el ascenso de otras naciones como China, aunque también juegan un papel importante Alemania y Rusia.

Lo anterior lleva a plantear la posibilidad de que el mundo actual se dirige hacia un orden multipolar con EUA jugando, por supuesto, un papel importante. Para el análisis de este proceso de transición resulta conveniente a manera de parangón, abordar el proceso de declinación de la hegemonía inglesa de principios del siglo Xx con la experiencia estadounidense en el siglo actual.

Para llevar a cabo tal fin, el trabajo se dividió en cinco secciones, en la primera se presenta el planteamiento teórico que sirve de base para el análisis; en la segunda sección se presentan los antecedentes el ascenso de EUA a la hegemonía mundial; en la tercera sección se presenta el legado de la segunda posguerra en la conformación del orden bipolar y de la hegemonía estadounidense en el bloque capitalista; mientras que en la cuarta sección se desarrolla un análisis del papel que viene desempeñando China en los indicadores mundiales seleccionados. La hipótesis de investigación consiste en el plantear que el ascenso de China al primer plano mundial tiene similitudes y profundas diferencias con el ascenso estadunidense de principios del siglo xx y que China tiene grandes limitaciones para convertirse en una hegemonía mundial absoluta como lo fue EUA. Por último, en la quinta sección, se presentan las conclusiones.

\section{INTRODUCCIÓN METODOLÓGICA}

Dada su complejidad, la extensión del tiempo histórico a tratar, así como la dispersión teórica y la limitación metodológica actual que aqueja a las ciencias histórico-sociales, es conveniente definir los principales conceptos e instrumentos metodológicos a usar en por lo menos tres puntos: i) el punto de partida sistémico-estructural; ii) las concepciones de periodización histórica, y iii) los criterios de ubicación de hegemonía y transición de la misma. 


\section{i) Aspectos sistémico-estructurales}

Siguiendo las definiciones de Marx, Gramsci y otros economistas y sociólogos no marxistas, se concibe al capitalismo industrial moderno como un sistema complejo, dinámico y abierto, integrado por diversos planos o niveles interdependientes, que deben diferenciarse de manera analítica para la mejor comprensión de los procesos históricos así como de las interacciones y modalidades de integración; esos planos o niveles son los siguientes:

a) Una estructura productiva (tecno-económica) formada por diversos componentes, como una base mercantil y mecanizada de producción de bienes y servicios, empresas capitalistas y trabajo asalariado; desarrollo de la ciencia, la tecnología y el sistema financiero; infraestructura básica, disponibilidad de energía y de transportes y comunicaciones a escala internacional; el conjunto de estos elementos se proyectan en la modalidad en que cada nación se inserta a la economía mundial y se analizarán con indicadores como la participación en el PIB mundial y manufacturero, la Inversión Extranjera Directa (IED), la participación en las exportaciones mundiales y en el nivel de endeudamiento público. Estas estructuras productivas conforman una unidad dinámica que se transforma a lo largo del tiempo a partir de la acumulación de capital, la extensión y la estructura del mercado, entre otros. El análisis de estos elementos es destacado en el estudio de la hegemonía por autores como Kennedy (1995) y servirán de base para analizar el proceso de auge y declinación económica de EUA.

b) Un entramado socio-institucional basado en la propiedad privada y en un trabajo asalariado libre de coerciones extraeconómicas, como principal base de sustentación de una estructura social más amplia y compleja consagrada en instituciones como el Estado.

c) Una cultura propia de cada nación basada en elementos históricos que generan diferentes grados de cohesión al interior de cada país.

Estos elementos de carácter superestructural los plantean Keohane (1984) y Gilpin(1987) al analizar el proceso de conformación de la hegemonía absoluta estadounidense en un país tan dominante que exporta sus instituciones (el liderazgo en la ONU, la OMC o el FMI y elementos esenciales de su cultura, como el american way of life). 


\section{ii) Periodización}

Para efectos de periodización, se utilizaron dos tipos distintos de instrumentos analíticos complementarios: 1) los cambios estructurales de las etapas del capitalismo a lo largo del tiempo, considerando que dichas etapas se traslapan en la transición de una a otra, dando lugar a cambios sustantivos que afectan a sus niveles y relaciones de articulación, y 2) la periodización del desenvolvimiento de la economía mundial visto a partir de los grandes cambios en la economía internacional que generan modificaciones en la hegemonía considerando para ello los planteamientos de Arrighi (1999) y Hobsbawm (2004 y 2013).

Se consideran las particularidades de las distintas etapas del capitalismo: el monopolista e imperialista clásico, el fordista-keynesiano o el capitalismo informático global actual (Dabat, 2017).

En lo que respecta a la periodización del desenvolvimiento de la economía mundial, los trabajos de Hobsbawm (2004 y 2013), Arrighi (1999) y Arrighi y Silver (2001) son de gran apoyo. Mientras Hobsbawm caracteriza al siglo xx como un periodo "corto" iniciando en 1914 y que concluye en 1991; Arrighi lo considera "largo" con fecha de inicio en 1873 y fecha de término a finales de la década de los ochenta del siglo xx.

Abundando, Hobsbawm observa que el inicio de la Primera Guerra Mundial marca el fin de una etapa del capitalismo conocida como capitalismo de libre concurrencia en el cual era clara la hegemonía inglesa; a partir de 1914 el mundo entró en un periodo de transición dando un importante papel a lo largo del "siglo corto" al Estado en la economía y EUA como país hegemónico. El orden bipolar emanado de la segunda posguerra será un elemento característico de este siglo, marcando su final con la caída del régimen soviético.

Por su parte, Arrighi plantea que la crisis económica de 1873 marcó el fin de un ciclo de prosperidad económica que propició el inicio del siglo $\mathrm{xx}$, ante lo cual la especulación financiera se convirtió en un elemento central como medida de sustitución ante la caída de la rentabilidad manufacturera y es hasta las crisis de la década de los noventa que se retoma el crecimiento del sector financiero que marcó el fin del siglo xx. A través del "Siglo largo", Arrighi y Silver observan la transición de la hegemonía inglesa a la estadounidense.

No obstante, ambos planteamientos resultan útiles para comparar el cambio de época de la economía mundial actual y de la crisis de hegemonía. El planteamiento del "siglo corto" de Hobsbawm es la mejor herramienta de análisis para el objetivo de este análisis porque en su planteamiento el siglo xx inicia justo con el ascenso de EUA al primer plano mundial, y concluye con 
el fin de la Guerra Fría en 1991; lo que sigue es un proceso complejo de gran crecimiento de la hegemonía estadounidense durante los años noventa, en lo que se considera la época de la hegemonía absoluta de EUA -que sólo duró 10 años-, pues para 2001 la crisis punto-com puso fin al periodo de expansión estadounidense, momento en que se desafió su poderío político y militar con el ataque del 11 de septiembre y emergió a escala mundial un nuevo rival gracias al ingreso de China a la omc.

\section{iii) Elementos que conforman la hegemonía}

El concepto de hegemonía implica necesariamente la comparación entre naciones. Un país determinado puede ser más fuerte que en el pasado, pero si ha sido rebasado por otro, no es hegemonía. En ese sentido, la hegemonía estadounidense de la segunda posguerra, sobre todo de la década de los noventa, se genera a partir de la conjunción del gran avance estadounidense junto con el declive de sus competidores: Alemania y Japón tras la Segunda Guerra Mundial y de la URSS en los años noventa del siglo pasado. De esta manera, se plantea un conjunto de indicadores basado en connotados estudiosos del tema que ayudan a evaluar el desempeño de las naciones hegemónicas.

De acuerdo con Kennedy (1995 y 1998), Keohane (1984), Gilpin (1987), Nye (2003) y Wallerstein (2007) se consideran grandes potencias a aquellos países que cuentan con: 1) extensión territorial y riqueza de sus recursos naturales, 2) poderío industrial, 3) poderío financiero, 4) liderazgo en ciencia y tecnología, 5) poderío político-militar, 6) liderazgo cultural, e 7) incidencia internacional a partir del sistema internacional de Estados o instituciones y organizaciones internacionales que tienen el poderío suficiente como para regir los destinos del mundo o a partes de él, sea por sí mismos o como parte de un sistema de grandes potencias.

El puro liderazgo económico, científico o militar no basta para hegemonizar al mundo, se requiere además que la cultura y las instituciones sean consideradas modelos a seguir en su mayoría por los países. Como se verá a continuación, el tránsito hacia la hegemonía estadounidense implicó todos los elementos considerados por el conjunto de autores seleccionados; sin embargo, en la actualidad, el avance chino carece de varios de los rubros planteados. 


\section{AGOTAMIENTO DE LA HEGEMONÍA INGLESA Y ASCENSO DE EUA}

La hegemonía inglesa basada en factores como la Revolución Industrial, su flota naval, el dominio del comercio internacional, el liderazgo monetario y financiero institucionalizado mediante el patrón oro, y el poderío militar entre otros, mostró señales de agotamiento desde finales del siglo XIx; en tanto que EUA se perfilaba como una nación cada vez más poderosa (Landes, 1979). Entre los factores de la decadencia británica se aprecia que su ventaja monopólica basada en su adelanto tecnológico fue disminuyendo a lo largo del siglo XIX. Naciones como EUA y Alemania consolidaron una fuerte base tecnoeconómica que competía en el liderazgo con Inglaterra. Como consecuencia, se observa una caída de la participación manufacturera inglesa (Kennedy, 1998); por ejemplo, para 1900 EUA ya superaba a Reino Unido en el potencial industrial y en la participación relativa de la manufactura a nivel mundial (véase cuadro 1).

En el aspecto financiero y bancario Inglaterra también sufrió una disminución de su poderío. La Gran Depresión (1870) y el pánico bancario (1890) generaron un importante incremento de la deuda externa de países como Argentina y Uruguay, en consecuencia, los bancos ingleses como el Baring tuvo que ser rescatado (Hobsbawm, 2013), o bien el caso del City of Glasgow Bank que atravesó por dificultades en 1878, o la caída del patrón oro de 1919 (Marichal, 2010).

En lo relativo a su poderío militar, se dan múltiples reveses al entonces poderoso ejército inglés. La ola de levantamientos coloniales iniciada con la rebelión maorí en Nueva Zelanda y el inicio de las luchas por la independencia de la India a mediados del siglo XIX marcaron el principio de otras derrotas en regiones importantes, como fue la rebelión de Jamaica, la guerra Zulú, el fracaso de la invasión a Afganistán, entre otras.

Por el contrario, en el caso de las potencias competidoras, EUA experimentó un ascenso importante desde finales del siglo XIX como consecuencia de la difusión de los adelantos tecnológicos ingleses a través del comercio internacional, de las inversiones inglesas y por la propia generación de universidades. EUA alcanzó el nivel de Reino Unido en materia económica, científica y tecnológica (Madisson, 2002). Si a esto se le suman las ventajas de territorio y recursos naturales de los estadounidenses se explica su ascenso a los primeros planos mundiales previo a la Primera Guerra. Para entonces, la capacidad tecnológica y productiva de EUA ya superaba a la del Reino Unido, lo cual se observa en los niveles de productividad y participación del PIв de ambos países a nivel mundial (véase cuadro 2). 


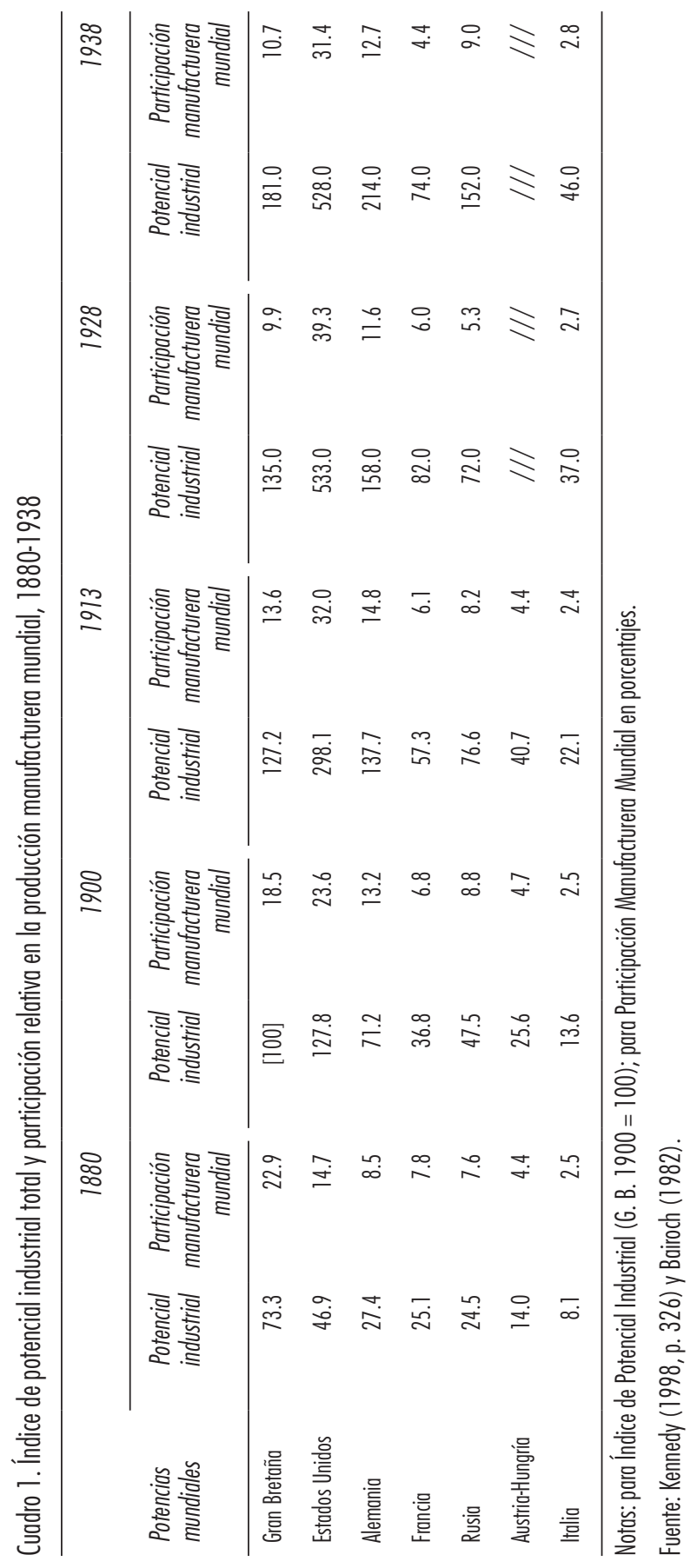




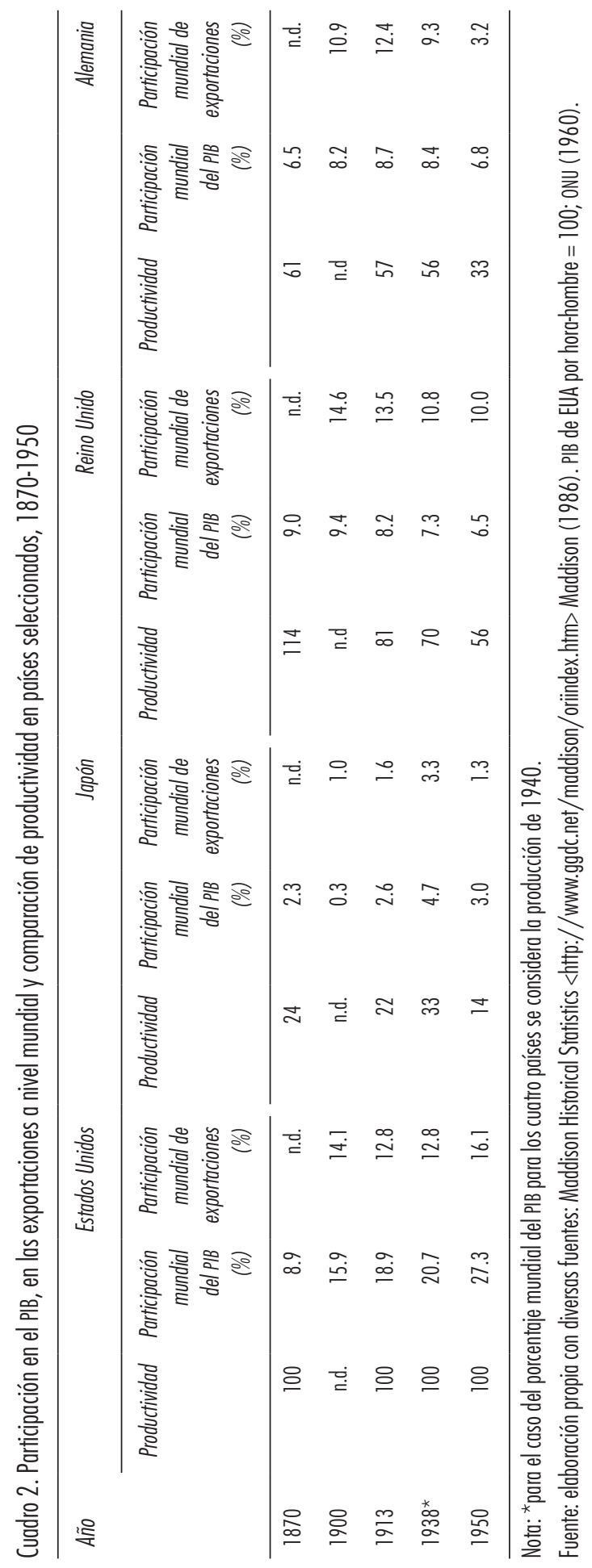


Sin embargo, el liderazgo inglés en materia monetaria y financiera, en la IED y en la participación en las exportaciones a nivel mundial se mantuvo debido a la madurez financiera y comercial de las empresas inglesas; pero también debido a la política aislacionista de EUA. El proceso de internacionalización de las empresas estadounidenses fue un fenómeno que se retrasó varias décadas por varias razones (la política aislacionista, la Primera Guerra, la crisis del 29 y la Segunda Guerra Mundial); será hasta después de la década de 1940 que las empresas estadounidenses se vuelquen hacia el mercado mundial.

El crecimiento estadounidense de finales del siglo xix y hasta 1913, estuvo concentrado en su mercado interno que se reforzaba con grandes inversiones extrajeras o importaciones de tecnología y maquinaria de Reino Unido y otros países europeos. Este fenómeno se correspondía a su vez con una débil y muy variable participación en aspectos mundiales, salvo en el concerniente al área geográfica que se refería más a sus intereses nacionales inmediatos.

Este carácter "autocentrado" del capitalismo estadounidense de aquella época, se debió a varios factores como el aislamiento geográfico en relación con Europa, la enorme amplitud territorial adquirida por anexión o compra de territorios vecinos, una población por completo incorporada a la economía mercantil a partir de una colonización agraria de inmigración europea y la venta de tierras a precios simbólicos; la enorme variedad y vastedad de sus recursos naturales; el papel fundamental del ferrocarril; el proteccionismo arancelario que pasó de 40 al 57\% entre 1861 y 1908; el vertiginoso crecimiento de la gran empresa y por la productividad del trabajo. Todo lo anterior conformó un mercado interno en constante crecimiento, una mayor rentabilidad capitalista que la de los países europeos, una fuerte atracción de capital extranjero que superaba a las exportaciones propias de capital y un entramado cultural e institucional muy acorde a las necesidades de reproducción del capitalismo (Weber, 2003).

El conjunto de estos elementos condujo a un muy particular tipo de inserción en las relaciones internacionales que, según Kennedy (1998) hizo que EUA fuera ya entonces considerada "una gran potencia" (la primera del mundo en términos industriales y de producto por habitante), a pesar de no ser todavía "parte del sistema de las grandes potenticas".

En conclusión, a inicios del siglo pasado, EUA tenía ya un vasto territorio geográfico -incluidas las anexiones de más de la mitad del territorio mexicano (1848), Florida (1819), Alaska (1867), Hawái (1898) y, por último, Puerto Rico (1917)-; contaba también con una gran dotación de recursos naturales, el más grande mercado interno, una importante tecnificación de la producción, tal vez de lo que carecía para ser "una gran potencia" mundial era su liderazgo 
en cuestión financiera y militar, pero el propio aislacionismo estadounidense impedía desarrollarlo. Fue la Primera Guerra Mundial la que serviría para empujar a la transición de la hegemonía, con otro actor importante, Alemania que contaba con un gran poder productivo y militar aunque carente del territorio suficiente para llegar a ser hegemonía (Frieden, 2007). Por otra parte, las mejores condiciones tecnológicas, productivas y de recursos naturales de EUA marcaban el inicio de la transición de la hegemonía. De acuerdo con Arrighi y Silver (2001), el crecimiento de la participación productiva de EUA en el PIB mundial tuvo como contracara el mayor poderío inglés en el sector financiero (situación similar a la que ocurre en la actualidad entre China y EUA). En ambos casos, como planteaba Braudel (1984), se está ante "la llegada del otońo" en la hegemonía mundial y el liderazgo monetario y financiero como último recurso para mantener un nivel de liderazgo mundial.

El estallido de la Primera Guerra Mundial derivó en la derrota de Alemania y Austria-Hungría, lo que frenó momentáneamente sus aspiraciones a convertirse en hegemonía mundial. La guerra tuvo consecuencias desastrosas para esos imperios. Alemania fue casi destruida y su población sufrió un golpe formidable. Los resultados de la Gran Guerra fueron también desbastadores para el conjunto de Europa a excepción de los países relativamente neutrales. En ese sentido, puede ser considerada como el primer gran capítulo de la autodestrucción de Europa para ser luego complementado por otro capítulo, el de la Segunda Guerra Mundial.

A estos eventos, se sumaron otras tres consecuencias geopolíticas y sociopolíticas fundamentales: la integración de la Unión Soviética, el primer gran salto de EUA en el camino por convertirse en gran superpotencia capitalista mundial y los comienzos de los movimientos nacionalistas de liberación nacional en el mundo periférico. El sistema monetario internacional centrado en el Banco de Inglaterra, se vio afectado -en tanto llegaba el fin del patrón oro-, que nunca pudo restablecerse después del impacto de la Gran Guerra.

A partir de esa Gran Guerra, EUA pasó a ser un gran exportador de capitales (Marichal, 2010), a través de préstamos a países de Europa en guerra o en reconstrucción, lo que lo convirtió en el gran acreedor y en el principal sustento económico de la recuperación del capitalismo europeo durante la década siguiente interrumpida por la Gran Crisis de 1929 como se verá a continuación.

Una de las grandes paradojas que siguió al fin de la Primera Guerra Mundial, fue el comportamiento de EUA frente a la realidad europea y mundial. Tras haber sido el principal artífice final de la derrota de Alemania y de la posterior creación de la Sociedad de las Naciones por iniciativa personal del 
presidente Wilson, EUA retornó al relativo aislamiento internacional por mandato de su propio Congreso (Brinkley, 2008). Ello condujo tanto a la autoexclusión estadounidense de la Sociedad de las Naciones como al restablecimiento de los altos niveles de proteccionismo comercial anterior (debido a la presión de la gran empresa norteamericana), hasta entonces poco involucrada en temas mundiales.

Este neoaislacionismo estadounidense coincidió con uno de los periodos de mayor crecimiento económico del país (más del $4 \%$ anual en los años veinte del siglo pasado) y relativo bienestar social, basado casi exclusivamente en el mercado interno y el laissez faire, bajo una impronta cultural conservadora y anticomunista alimentada por la agitación antibolchevique de los medios de comunicación. La base tecnoeconómica del auge fue el primer despliegue del fordismo como fenómeno dominante, junto a la innovación tecnológica permanente, y a un sistema bancario y de crédito moderno que financió la elevación de la demanda, aunque sólo a un nivel nacional.

Dada la declinación de Europa, EUA alcanzó entonces una enorme superioridad económica mundial (de implantación del fordismo 30 años antes que Europa Occidental o Japón), sin que ello se expresara en hegemonía mundial. Pero este proceso fue interrumpido por la Gran Crisis de 1929 y la posterior depresión que afectó, sobre todo, al sistema financiero y dio lugar a una gran depresión en el país que se trasladó de inmediato a Europa poniendo fin a su breve recuperación de 1925-1929, y que abrió paso a las reformas del New Deal.

Entre la Gran Crisis de 1929 y la Segunda Guerra Mundial, como resultado de los fenómenos expuestos, el mundo volvió a presenciar grandes cambios: 1) se acentuó el proteccionismo comercial e inició el control de cambios, en un contexto de declinación del comercio internacional y de los movimientos internacionales de capital (Pinder, 1976); 2) tuvo lugar la rápida industrialización rusa basada en la industria pesada, en un proceso paralelo al despliegue de la Revolución china; 3) bajo el nazismo, Alemania construyó una poderosa economía de guerra contra la Unión Soviética y los triunfadores europeos de la Primera Guerra Mundial; 4) comenzó el crecimiento acelerado de la economía japonesa y la expansión imperialista del país hacia Manchuria, China, el Pacífico y las colonias francesas, inglesas y holandesas del sudeste, y 5) inició la industrialización sustitutiva en América Latina, destacando los casos de México, Brasil y Argentina.

En 1939 comenzó la Segunda Guerra Mundial con la invasión a Polonia por la Alemania nazi. De hecho, la Segunda Guerra Mundial debe ser vista como una continuación de la primera contienda bélica, en cuanto al 
intento de Alemania por lograr la hegemonía mundial ahora con el apoyo de Italia y Japón, disputándole el liderazgo con el debilitado Reino Unido y ulteriormente a EUA. La nueva guerra tuvo un alcance territorial más amplio (incluyó a Asia Oriental, al Pacífico y a África del norte, además de Europa) y un contenido más sociopolítico e ideológico, porque contrapuso al fascismo, al comunismo y a la democracia liberal, y se basó en el empleo de armas mucho más poderosas y mortíferas provocando impactos más letales, cobró más víctimas humanas y una enorme destrucción de infraestructura física y económica tanto en Europa (sobre todo en Alemania y la URSS) como en Asia Oriental, sobre todo en Japón por las bombas nucleares.

El elemento común en ambas guerras fue, junto al declive de Europa, el ascenso de EUA a la hegemonía mundial y el ascenso del campo socialista al primer plano creando un orden bipolar con la Unión Soviética primero, y luego con China, aliada en un inicio a la primera. Este orden permaneció vigente hasta la década de 1990, cuando EUA vivió un segundo gran momento de su hegemonía mundial (véase figura 1), para luego caer en descomposición a partir de 2001 con la crisis punto-com y el ascenso de China en el panorama mundial.

\section{LA SEGUNDA POSGUERRA Y EL NUEVO ORDEN MUNDIAL}

A partir de los logros sumados en ambas guerras, EUA pasó a encabezar la hegemonía mundial por su enorme fuerza económica, financiera, política y militar. Para 1945 tenía un producto bruto 60\% superior al total de las otras 15 economías más fuertes del planeta (Marichal, 2010), dos terceras partes de la capacidad industrial y tres cuartas partes del capital invertido en el mundo (Hobsbawm, 2004), a los que se le sumaba su incomparable capacidad científica, tecnológica, empresarial y financiera. Cabe aclarar que el resto de las naciones importantes en el mundo capitalista quedaron devastadas tras las dos guerras, por lo que la hegemonía estadounidense fue total y prueba de ello fue la imposición del dólar como única moneda líder a nivel mundial.

A diferencia de lo sucedido tras la Primera Guerra Mundial, la entrada dominante de EUA en el escenario mundial fue definitiva, tanto por los nuevos intereses económicos mundiales de su capitalismo, como por el desafío comunista.

La Unión Soviética también se había convertido en gran potencia económica, política y militar, basada en una poderosa industria pesada y, desde 
1949, en su potencia nuclear. Esto dio lugar a la nueva relación mundial de fuerzas del mundo bipolar de la Guerra Fría basada en el equilibrio nuclear, por encima de la autoridad formal de la ONU creada tras la guerra.

Para contener la expansión mundial del comunismo, EUA construyó un cerco de resguardo en torno al nuevo campo socialista en Europa, el Plan Marshall, la OCDE o la OTAN fueron junto a los primeros basamentos de la Unión Europea, instrumentos del relanzamiento de Europa Occidental. En Asia lo fue Japón y demás aliados de Asia Oriental y los futuros "tigres asiáticos", o Israel en el Oriente Medio más tarde. Tales políticas subsistieron hasta que la recuperación capitalista de estos países los convirtió en fuertes competidores de EUA. ${ }^{1}$

La recuperación de Europa Occidental dio lugar al denominado "Estado de Bienestar" (Maddison, 1986; Hobsbawm, 2004), resultante de una recuperación mundial que también incluyó gran parte del Tercer Mundo. ${ }^{2}$ Con esto EUA confirmó su papel de hegemonía liderando las instituciones que generaron el orden mundial hasta nuestros días: los acuerdos de Bretton Woods que le otorgaron el liderazgo monetario, pero también instituciones como: la ONU, la omc, la otAn, el Banco Mundial, el FMI, la OCDE, entre otros.

Sin embargo, aun con las instituciones que regulan el orden mundial de su lado, EUA ha tenido tropiezos económicos y crisis de su hegemonía. La expansión del capitalismo de la posguerra se realizó en dos etapas separadas por la crisis mundial de 1974-1975 y la subsiguiente recesión. La primera fue la de expansión mundial generalizada que culminó en la crisis mundial de 19741975 provocada por el agotamiento del fordismo-keynesiano y el subsiguiente estancamiento inflacionario de EUA, que llevó a la inconvertibilidad del oro en dólar y el fin del sistema monetario de Bretton Woods, en un contexto de derrumbe de la rentabilidad capitalista.

La segunda etapa expansiva comenzó hacia fines de los años ochenta, con la irrupción del capitalismo informático y la globalización encabezada por EUA bajo el neoliberalismo. Pero este proceso fue muy breve (no trascendió a la última década del siglo) como resultado de la acentuación de los aspectos

1 El orden mundial de posguerra se basó en dos pilares básicos: a) los acuerdos BrettonWoods de 1944 (no ratificados por la Unión Soviética), de creación de un sistema monetario basado en el dólar convertible a oro como moneda mundial junto a un sistema de paridades monetarias fijas supervisado por el FMI; y b) la onU creada un año después (1945), que reconocerá de hecho el nuevo papel de la Unión Soviética como miembro del Consejo de Seguridad con derecho a veto.

2 En América Latina y otras partes del Tercer Mundo, la prosperidad de fines de los años sesenta y de la década siguiente fue un fenómeno engańoso, por basarse en el creciente endeudamiento masivo e insostenible que condujo poco después, a la Gran Crisis de la deuda. 
nocivos del neoliberalismo como la sobre especulación financiera y su política de neoimperialismo globalista. ${ }^{3}$

Al contrario de lo sucedido con EUA y los países capitalistas desarrollados, la URSS se vio afectada por los cambios de la economía mundial de los años ochenta. No sólo dejó de crecer a tasas superiores a las de los países capitalistas, sino que su estatismo burocrático entró en una etapa de declinación irreversible. Esta involución coincidió con el inicio de la recuperación del capitalismo estadounidense y europeo, resultante de la revolución informática y su liderazgo en la globalización, para dar lugar al colapso de la URSS y de su entorno de Europa Oriental a principios de la década de los noventa del siglo xx; para Hobsbawm este colapso marcó el fin del "corto siglo xx".

Como se puede observar, en la década de los noventa del siglo pasado, el deterioro del bloque soviético dejó a EUA como única súper potencia mundial y el fortalecimiento de éste a partir de ser el epicentro de la revolución informática, le dio una gran ventaja en términos científicos, tecnológicos, comerciales, militares y culturales; la hegemonía absoluta de EUA tuvo su esplendor durante la década de 1991 a 2001 y fue su carácter especulativo, cortoplacista e individualista, el que generó las condiciones de agotamiento de su crecimiento económico para decantar en la crisis de 2001. En lo político y militar ha sido cuestionado su liderazgo con los atentados del 11 de septiembre y su derrota en Oriente Medio.

En el caso de China, este país emprendió un ascenso a partir de su planteamiento de "socialismo de mercado", resultado de un largo y complejo proceso de grandes pugnas internas que culminaron a mediados de los años setenta con el triunfo de la línea pragmática de industrialización moderna e integración al mercado mundial de Deng Xiaoping. ${ }^{4}$ El resultante fue una economía mixta dirigida por el Partido Comunista Chino, con un sector público dominante sobre múltiples tipos de empresa (estatales, transnacionales, mixtas, privadas, cooperativas, campesinas, municipales, etcétera) y a partir

Desplazamiento hacia el exterior de su producción industrial, desregulación financiera especulativa, militarización de su política exterior, consumismo excesivo o desgarramiento su propio tejido social interno.

4 La era maoísta había abierto las puertas a la educación básica, la salud pública y a la tecnología mejorada. La doctrina del igualitarismo había otorgado al campesino una nueva visión de sí mismo y sus potencialidades. La suma de la anterior revolución agraria y las posteriores reformas modernizadoras, permitieron la creación de un enorme mercado interno que, décadas después, en plena crisis internacional, haría posible la reorientación "hacia adentro" de la economía china, sin desmedro significativo de su crecimiento (Dabat y Leal, 2013). 
de 2001 una inserción competitiva en la globalización basada en el aprendizaje tecnológico y la innovación, que ha llevado a la emergencia tendencial de una nueva bipolaridad mundial y su consecuente crisis de la hegemonía estadounidense.

\section{LA CRISIS DE HEGEMONÍA ACTUAL}

El ascenso de los dos nuevos polos no ocurrió en forma lineal. La conversión de EUA en superpotencia hegemónica del capitalismo atravesó tanto por etapas de aislamiento internacional relativo, como de involucramiento total en los asuntos internacionales, así como por prolongadas oleadas de ascenso (segunda posguerra y década de los noventa del siglo $\mathrm{xx}$ ) y caídas (crisis en las décadas de los treinta, los setenta y parte de los ochenta del siglo xx, véase figura 1). El planteamiento del presente artículo es que desde la crisis puntocom, EUA se encuentra sumido en un proceso de declive de su hegemonía del cual difícilmente se recuperará, una etapa similar a la ocurrida desde finales del siglo xIX con la hegemonía inglesa (véase figura 1).

Figura 1. Momentos en la transición de la hegemonía mundial

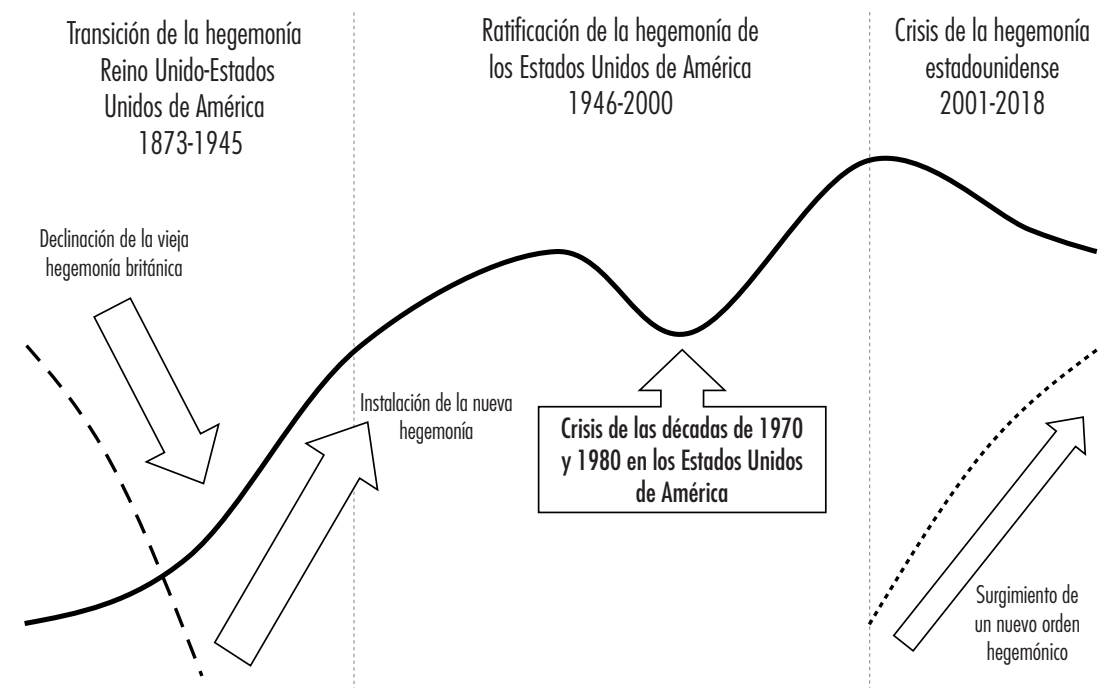

Fuente: Arrighi y Silver (2001); Hobsbwam (2004); Dabat y Leal (2013); Dabat et al. (2012). 
De manera similar, al ascenso de EUA al primer plano mundial en esa época, hoy se observa el ascenso al primer plano mundial de China y en menor medida de otros países como Alemania, Rusia o Corea, lo que al parecer conforma una nueva etapa de transición de la hegemonía mundial.

El caso chino es un fenómeno complejo y de larga duración. Tras ser una primera potencia del mundo hasta el siglo Xv, pasó a ser un Imperio conservador decadente, semicolonizado por las potencias imperialistas en el siglo XIX hasta el borde de la partición territorial. Posteriormente, basado en un fuerte movimiento nacional y revolucionario, se convirtió en la gran potencia que es en la actualidad, aprovechando su amplia mano de obra y sus recursos naturales para la generación de manufacturas, incrementando su presencia en el mundo a partir de una estrategia de exportaciones y de IED.

El 2001 resulta ser central en el proceso de declinación de la hegemonía absoluta de EUA, pues marca el fin de su fase de expansión económica; el ataque terrorista de septiembre de ese año fue un claro desafío a su hegemonía militar y la entrada de China a la OMC conformó en conjunto una serie de hechos históricos que marcaron el inicio del proceso de transición hegemónica.

La salida de la crisis punto-com se basó en la caída de las tasas de interés y el crecimiento de la deuda pública con el objetivo de reanimar su economía, en los hechos lo lograron, pero mediante la generación de una burbuja financiera (inmobiliaria) similar a la que ocurrió a finales del siglo xIx y principios del xx durante la crisis de la hegemonía inglesa.

Dicha burbuja se derrumbó en 2008 y dio paso a la crisis de 2009, desde entonces, EUA se encuentra en una profunda recesión que ha ido aminorando gracias a la política de flexibilización monetaria (quantitative easing) que estableció las tasas de interés cerca del $0 \%$. Si bien se han elevado gradualmente, EUA se encuentra en una situación similar a la que Keynes denominó trampa de la liquidez, y que consiste en tasas de interés bajas que no generan un crecimiento importante en la inversión productiva ni en el empleo a pesar de la excesiva liquidez que existe en el mundo, y sí generan un incremento del atesoramiento y de la incertidumbre mundial que no generan crecimiento económico fuerte y sí endeudamiento (véase cuadro 4).

En el caso de China, se han mantenido tasas sostenidas de crecimiento del PIB, de las exportaciones y de las manufacturas en todo este periodo de debacle estadounidense (similar a lo ocurrido entre 1870 y 1928 entre EUA y el Reino Unido, véanse cuadros 1 y 2).

Hacia fines de esta década se observa que el proceso de transformación de la economía mundial está más acelerado que nunca y es importante mirar atrás para dimensionar la importancia de los cambios que se presentan. Al 
respecto, el propio Global Trends (2012), organismo gubernamental estadounidense, considera que para 2030 EUA no será más el país hegemónico que dirija los destinos del mundo como lo hizo tras la Segunda Guerra Mundial, ya que tendrá que compartir la hegemonía mundial con China, el cual ya habría rebasado por mucho a EUA en lo económico. Sin embargo, no existirá un país que tenga las condiciones de ejercer una hegemonía absoluta como lo hizo EUA, por lo que el mundo se dirigirá hacia un orden multipolar. ${ }^{5}$

Como se puede ver en el cuadro 4, EUA sigue teniendo una importante superioridad científica-tecnológica y empresarial, aunque China se destaca no sólo por la vastedad de sus procesos de aprendizaje tecnológico popular, sino además, por la mayor orientación de ésta a fines civiles y sociales (Dabat y Leal, 2013). Habría que agregar al respecto que la vía china está aún muy limitada, por su falta de apertura política y otros requerimientos sociales, institucionales, culturales y ambientales; aunque tampoco EUA se encuentra para nada adelantada, a pesar de su discurso triunfalista.

El ascenso de EUA a la hegemonía mundial se encuentra hoy en un proceso importante de cambio, en el cual existe un debate abierto sobre el destino final de la hegemonía mundial estadounidense. Frente a este debate, la preocupación central no es tanto medir con precisión el actual nivel de declinación de EUA, sino más bien, aproximarse histórica y cualitativamente al problema a través de la interrelación de los siete indicadores planteados, como del análisis de las posibilidades históricas del país para preservar su papel hegemónico mundial ante el ascenso de China y otras naciones emergentes del mundo periférico.

En este sentido, se parte de la idea de que, aunque EUA sigue siendo la principal potencia mundial, lo es de forma decreciente por diversas razones, entre las que destacan: la posición del país dentro de la división internacional del trabajo (su posición cada vez menos productiva y competitiva) derivada tanto de sus condiciones internas (tecno-productivas, institucionales, y sociales), como de su falta de capacidad competitiva a nivel mundial y de su falta de capacidad para sostener su poder hegemónico (Dabat y Leal, en prensa).

5 Global Trends 2030 es un foro patrocinado por el National Intelligence Council, organismo gubernamental de EUA, en el que se discuten bajo invitación los temas que abordarán las publicaciones encargadas de realizar estimaciones imparciales y alejadas de los objetivos coyunturales de la política implementadas por el gobierno estadounidense. Estas estimaciones son utilizadas por la Oficina del Director de Inteligencia Nacional y el Consejo Nacional de Inteligencia de EUA en la formulación de políticas enfocadas a la planificación a largo plazo en temas de importancia mundial como la globalización, la demografía y el medio ambiente. En su última edición 2012, se plantea que para 2030 el avance de China, más el debilitamiento de EUA, llevarán al mundo hacia el fin de la hegemonía absoluta de EUA y hacia el advenimiento de un mundo multipolar. 
La incertidumbre que genera las posibles resoluciones respecto a políticas comerciales del presidente Donald Trump, el reconocimiento del desgaste de su infraestructura, el envejecimiento de su población, la declinación de su liderazgo en Medio Oriente, entre otros factores, hace plantear que el mundo se dirige hacia un orden multipolar con China, Rusia, Alemania y otras naciones jugando un papel importante de contrapeso. De manera similar a lo ocurrido a principio del siglo xx con EUA, China lidera al mundo en varios indicadores económicos (véase cuadro 3).

Sin embargo, más allá del análisis productivo y manufacturero, se observan algunas ventajas y rezagos de EUA en los indicadores de la hegemonía mundial (véase cuadro 4).

Cuadro 3. Comparativo de indicadores seleccionados EUA y China, 2000-2017

\begin{tabular}{|c|c|c|c|c|c|c|c|c|}
\hline \multirow[t]{2}{*}{ Año } & \multicolumn{2}{|c|}{$\begin{array}{r}\text { Participación en el PIB } \\
\text { mundial PPP* }\end{array}$} & \multicolumn{2}{|c|}{$\begin{array}{r}\text { Participación en } \\
\text { la producción } \\
\text { manufacturera mundial }\end{array}$} & \multicolumn{2}{|c|}{$\begin{array}{r}\text { Participación en las } \\
\text { exportaciones a nivel } \\
\text { mundial }\end{array}$} & \multicolumn{2}{|c|}{$\begin{array}{r}\text { Reservas } \\
\text { Internacionales } \\
\text { (millones de dólares) }\end{array}$} \\
\hline & $\begin{array}{r}\text { Estados } \\
\text { Unidos de } \\
\text { América \% }\end{array}$ & $\begin{array}{r}\text { China } \\
\%\end{array}$ & $\begin{array}{r}\text { Estados } \\
\text { Unidos de } \\
\text { América \% }\end{array}$ & $\begin{array}{r}\text { China } \\
\%\end{array}$ & $\begin{array}{r}\text { Estados } \\
\text { Unidos de } \\
\text { América \% }\end{array}$ & $\begin{array}{r}\text { China } \\
\%\end{array}$ & $\begin{array}{r}\text { Estados } \\
\text { Unidos de } \\
\text { América }\end{array}$ & China \\
\hline 2000 & 21.2 & 7.6 & 25.4 & n.d. & 11.6 & 3.9 & 128 & 172 \\
\hline 2001 & 20.9 & 8.1 & 25.7 & n.d. & 11.3 & 4.3 & 130 & 220 \\
\hline 2002 & 20.6 & 8.5 & 25.3 & n.d. & 10.3 & 5.0 & 158 & 298 \\
\hline 2003 & 20.5 & 9.1 & 23.7 & n.d. & 9.2 & 5.8 & 184 & 416 \\
\hline 2004 & 20.1 & 9.5 & 22.4 & 8.7 & 8.5 & 6.4 & 190 & 623 \\
\hline 2005 & 19.9 & 10.1 & 22.0 & 9.6 & 8.3 & 7.3 & 188 & 831 \\
\hline 2006 & 19.2 & 10.7 & 21.6 & 10.8 & 8.2 & 8.0 & 221 & 1081 \\
\hline 2007 & 18.6 & 11.6 & 19.7 & 12.3 & 8.0 & 8.7 & 278 & 1546 \\
\hline 2008 & 17.8 & 12.2 & 17.8 & 14.6 & 7.8 & 8.9 & 294 & 1966 \\
\hline 2009 & 17.3 & 13.4 & 18.4 & 17.4 & 8.3 & 9.6 & 404 & 2453 \\
\hline 2010 & 16.8 & 14.0 & 17.3 & 18.4 & 8.3 & 10.3 & 489 & 2910 \\
\hline 2011 & 16.3 & 14.7 & 16.1 & 20.7 & 8.0 & 10.4 & 537 & 3255 \\
\hline 2012 & 16.1 & 15.3 & 16.4 & 22.5 & 8.3 & 11.1 & 574 & 3388 \\
\hline 2013 & 15.8 & 15.9 & 16.5 & 24.1 & 8.3 & 11.7 & 449 & 3880 \\
\hline 2014 & 15.7 & 16.5 & 16.5 & 25.3 & 8.5 & 12.4 & 434 & 3900 \\
\hline
\end{tabular}


Ascenso y declive de Estados Unidos en la hegemonía mundial

\begin{tabular}{|c|c|c|c|c|c|c|c|c|}
\hline \multirow[t]{2}{*}{ Año } & \multicolumn{2}{|c|}{$\begin{array}{r}\text { Participación en el PIB } \\
\text { mundial PPP* }\end{array}$} & \multicolumn{2}{|c|}{$\begin{array}{r}\text { Participación en } \\
\text { la producción } \\
\text { manufacturera mundial }\end{array}$} & \multicolumn{2}{|c|}{$\begin{array}{r}\text { Participación en las } \\
\text { exportaciones a nivel } \\
\text { mundial }\end{array}$} & \multicolumn{2}{|c|}{$\begin{array}{r}\text { Reservas } \\
\text { Internacionales } \\
\text { (millones de dólares) }\end{array}$} \\
\hline & $\begin{array}{r}\text { Estados } \\
\text { Unidos de } \\
\text { América \% }\end{array}$ & $\begin{array}{r}\text { China } \\
\%\end{array}$ & $\begin{array}{r}\text { Estados } \\
\text { Unidos de } \\
\text { América \% }\end{array}$ & $\begin{array}{r}\text { China } \\
\%\end{array}$ & $\begin{array}{r}\text { Estados } \\
\text { Unidos de } \\
\text { América \% }\end{array}$ & $\begin{array}{r}\text { China } \\
\%\end{array}$ & $\begin{array}{r}\text { Estados } \\
\text { Unidos de } \\
\text { América }\end{array}$ & China \\
\hline 2015 & 15.7 & 17.1 & 17.7 & 26.6 & 9.0 & 13.8 & 384 & 3405 \\
\hline 2016 & 15.4 & 17.7 & 17.6 & 26.2 & 8.9 & 13.1 & 406 & 3098 \\
\hline 2017 & 15.2 & 18.3 & n.d. & n.d. & 8.6 & 12.8 & 451 & 3236 \\
\hline
\end{tabular}

Notas: *se refiere al PIB medido como paridad de poder adquisitivo, PPP por sus siglas en inglés.

Fuente: elaboración propia con datos de Banco Mundial (2019) y OMC (2019), Series de tiempo desde 2000.

Cuadro 4. Indicadores seleccionados para la consolidación de la hegemonía 2018

\begin{tabular}{|c|c|c|c|c|}
\hline & & $\begin{array}{r}\text { Estados Unidos de } \\
\text { América }\end{array}$ & China & Alemania \\
\hline \multirow[t]{3}{*}{ Inversión } & Entradas de IED (posición a nivel mundial) & 1 & 2 & 11 \\
\hline & Salidas de IED (posición a nivel mundial) & 1 & 3 & 5 \\
\hline & $\begin{array}{l}\text { Número de patentes registradas por } \\
\text { residentes }\end{array}$ & 293904 & 1245709 & 47785 \\
\hline \multirow[t]{6}{*}{ Territorio } & Extensión territorial en $\mathrm{Km}^{2}$ & 9833517 & 9596960 & 357022 \\
\hline & Número de aeropuertos & 13513 & 507 & 539 \\
\hline & Km de las vías férreas & 226427 & 91000 & 41896 \\
\hline & $\begin{array}{l}\text { Número de embarcaciones de la marina } \\
\text { mercante }\end{array}$ & 3692 & 4610 & 629 \\
\hline & $\begin{array}{l}\text { Reservas probadas de petróleo } \\
\text { (posición a nivel mundial) }\end{array}$ & 12 & 14 & 49 \\
\hline & $\begin{array}{l}\text { Reservas probadas de gas natural } \\
\text { (posición a nivel mundial) }\end{array}$ & 203 & 9 & 64 \\
\hline \multirow[t]{3}{*}{$\begin{array}{l}\text { Sistema } \\
\text { Financiero }\end{array}$} & $\begin{array}{l}\text { Número de grandes bancos } \\
\text { (Top } 100 \text { a nivel mundial) }\end{array}$ & 15 & 16 & 1 \\
\hline & Número de bolsas de valores & 2 & 2 & 1 \\
\hline & $\begin{array}{l}\text { Partición en las reservas del } \\
\text { Banco Mundial }\end{array}$ & $16 \%$ & $5 \%$ & $3 \%$ \\
\hline
\end{tabular}


Cuadro 4. Indicadores seleccionados para la consolidación de la hegemonía 2018 (continuación)

\begin{tabular}{|c|c|c|c|c|}
\hline & & $\begin{array}{r}\text { Estados Unidos de } \\
\text { América }\end{array}$ & China & Alemania \\
\hline \multirow[t]{4}{*}{$\begin{array}{l}\text { Sistema } \\
\text { Financiero }\end{array}$} & $\begin{array}{l}\text { Participación en la Cesta de Monedas } \\
\text { del Fondo Monetario Internacional }\end{array}$ & $17 \%$ & $2 \%$ & $6 \%$ \\
\hline & $\begin{array}{l}\text { Entrada de Inversiones de Cartera } \\
\text { (Miles de millones de dólares) }\end{array}$ & 354828 & 168224 & 77983 \\
\hline & Volumen de la deuda pública (\% PIB) & $106 \%$ & $50 \%$ & $61 \%$ \\
\hline & $\begin{array}{l}\text { Reservas internacionales totales } \\
\text { (Millones de dólares) }\end{array}$ & 451 & 3236 & 199 \\
\hline \multirow[t]{5}{*}{$\begin{array}{l}\text { Ciencia y } \\
\text { Tecnología }\end{array}$} & $\begin{array}{l}\text { Número de personas dominan el idioma } \\
\text { a nivel mundial }\end{array}$ & $\begin{array}{r}\text { Inglés } \\
379 \text { millones } \\
\text { de personas }\end{array}$ & $\begin{array}{r}\text { Mandarín } \\
1311 \text { millones } \\
\text { de personas }\end{array}$ & $\begin{array}{r}\text { Alemán } \\
76 \text { 1millones } \\
\text { de personas }\end{array}$ \\
\hline & $\begin{array}{l}\text { Número de universidades de prestigio } \\
\text { (Top } 500 \text { a nivel mundial) }\end{array}$ & 146 & 44 & 39 \\
\hline & Premios Nobel & 377 & 8 & 107 \\
\hline & Número de publicaciones científicas por año & 408985 & 426165 & 103122 \\
\hline & Gasto en I+D (\% PIB) & $2 \%$ & $3 \%$ & $3 \%$ \\
\hline \multirow[t]{6}{*}{ Cultura } & $\begin{array}{l}\text { Producción de la industria cinematográfica } \\
\text { (Número de películas producidas) }\end{array}$ & 660 & 874 & 233 \\
\hline & $\begin{array}{l}\text { Ingresos industria cinematográfica } \\
\text { (Millones de dólares) }\end{array}$ & 19041 & 8595 & 2500 \\
\hline & $\begin{array}{l}\text { Producción industria musical } \\
\text { (Posición a nivel mundial) }\end{array}$ & 1 & 20 & 3 \\
\hline & $\begin{array}{l}\text { Ingresos industria musical } \\
\text { (Millones de dólares) }\end{array}$ & 4898 & 292 & 1404 \\
\hline & Producción industria de videojuegos & 2 & 1 & 5 \\
\hline & $\begin{array}{l}\text { Ingresos industria de los videojuegos } \\
\text { (Millones de dólares) }\end{array}$ & 31535 & 34400 & 4989 \\
\hline \multirow[t]{4}{*}{ Poder Militar } & Bases militares & 800 & 7 & 0 \\
\hline & Tropas activas & 1.3 Millones & 2.2 Millones & 178641 \\
\hline & Aeronaves militares & 13398 & 3187 & 613 \\
\hline & Embarcaciones militares & 415 & 714 & 81 \\
\hline $\begin{array}{l}\text { Poder Político } \\
\text { Internacional }\end{array}$ & $\begin{array}{l}\text { Participación en instituciones } \\
\text { internacionales }\end{array}$ & $\begin{array}{r}\text { G-5, G-7, G-8 } \\
\text { G-10, G-20, OCDE, } \\
\text { Interpol, 0NU, OTAN }\end{array}$ & $\begin{array}{r}\text { G-5 G-20, G-77, } \\
\text { ONU, Interpol, BRICS }\end{array}$ & $\begin{array}{r}\text { G-5, G-7, G-8 } \\
\text { G-10, G-20, 0CDE, } \\
\text { Interpol, ONU, OTAN, } \\
\text { Unión Europea }\end{array}$ \\
\hline
\end{tabular}




\begin{tabular}{llccrr}
\hline & & $\begin{array}{r}\text { Estados Unidos de } \\
\text { América }\end{array}$ & China & Alemania \\
\hline $\begin{array}{l}\text { Poder Político } \\
\text { Internacional }\end{array}$ & $\begin{array}{l}\text { Participación en el Consejo de Seguridad } \\
\text { de la ONU } \\
\text { Número de representaciones en el exterior }\end{array}$ & Permanente & Permanente & No permanente \\
& 273 & 268 & 224 \\
\hline
\end{tabular}

Fuente: CIA (2019); Arias (2019); Bankinter (2016); Banco Mundial (2019 y 2018); UnCTAD (2019); Eberhard et al. (2019); Expansión (2019); FMI (2019); Global Fire Power (2019); ONU (2019); Shangai Ranking Consultancy (2015); Statista (2019a); Statista (2019b); TradeMap (2019); unESCO (2019); Voz Libre (2018); YouBiolt.com (2019).

Como se puede observar en el cuadro 4, EUA sigue siendo el principal receptor y emisor de IED, aunque el ascenso de China es muy fuerte y cuenta con un nivel de endeudamiento muy inferior al de EUA. En el análisis territorial, EUA es una potencia en cuanto a extensión, población y recursos energéticos; mientras China le disputa el liderazgo en muchos de esos rubros, Alemania no figura, por lo que difícilmente será una hegemonía mundial y sólo participará como líder regional. En este rubro Rusia es un país importante, pero rezagado en los demás indicadores, por lo que sólo participará como líder regional.

La infraestructura aeroportuaria y ferroviaria es muy superior en EUA, pero China avanza de forma importante.

El liderazgo monetario y financiero de EUA se mantiene gracias al dólar y su profundización financiera, aunque China cuenta con bancos líderes a nivel mundial (Industrial and Commercial Bank of China 2o lugar y Banco de Construcción de China 5o lugar). EUA marcha a la cabeza en Ciencia y Tecnología, respaldado por sus grandes universidades y su liderazgo en microprocesadores. Aunque en las más recientes innovaciones (robótica, nanotecnología, adelantos médicos, biología sintética, impresoras 3D, entre otras) EUA figura en los primeros lugares, China, Alemania, Rusia, Corea, entre otros países, comparten el liderazgo en innovación, lo que implica que difícilmente EUA podría reeditar el liderazgo mundial de la revolución informática que lo llevó a los primeros planos en la década de los noventa. EUA sigue siendo la principal potencia militar, Alemania está muy lejos de serlo, y China al parecer también. Rusia si bien podría ser una potencia importante, no cuenta con otros elementos como ya se mencionó líneas arriba.

En lo relacionado con la exportación de la cultura, las películas más vistas son las producidas en Hollywood, los artistas más reconocidos en el ámbito internacional son los angloparlantes. Se puede concluir que EUA sigue manteniendo un liderazgo cultural. Y finalmente, en lo relativo al orden institucional a nivel mundial, destaca la preeminencia de EUA en las principales 
organizaciones como en el Consejo de Seguridad de la ONU, el FMI, la OMC, el G7. Dichas organizaciones se crearon tomando como modelo la democracia liberal representativa promovida sobre todo por EUA, así se vislumbra difícil que las instituciones y las prácticas chinas (como la dictadura partidista, la falta de elecciones o la censura de Internet) puedan exportarse a nivel mundial y se conviertan en modelo a seguir aunque representen una organización de un país estable y con mejoras sociales.

Por todo lo anterior, se considera que el liderazgo de EUA se encuentra en un proceso de disminución en prácticamente todos los rubros, lo que lo imposibilita seguir ejerciendo una hegemonía absoluta, pero ni China ni algún otro país están en condiciones de ejercerla. Por lo que de nuevo se reafirma que se avanza hacia un mundo multipolar.

Lo que diferencia a la nueva polarización del mundo de las anteriores formas de configuración del orden mundial, es que ahora no se basa en dos grandes bloques cerrados o semicerrados entre sí (o en la pluralidad de múltiples espacios colonial-imperialistas igualmente cerrados o semicerrados que la precedió), sino dentro del espacio abierto de la globalización y la interdependencia comercial y financiera entre países. Ello hace que el balance de poder entre las dos grandes potencias dependa en principio (no puede descartarse la posibilidad de una nueva Guerra Mundial por completo devastadora) del ritmo y la calidad del desarrollo interior, así como de las vinculaciones y alianzas exteriores, en la cual las relaciones de China con los países en desarrollo emergentes le otorgan ventaja.

\section{CONCLUSIONES}

La metodología planteada resultó muy útil para analizar el proceso histórico de ascenso de EUA a la hegemonía mundial, en ese sentido, se observó que desde antes de la Segunda Guerra Mundial EUA ya contaba a nivel mundial con un desempeño importante en rubros como la participación en el PIB, en el comercio internacional, los flujos financieros y la productividad; era pues un país importante en la generación de innovaciones y sus universidades eran importantes a nivel mundial. De la misma forma, en la actualidad, China es el primer productor de manufacturas del mundo, tiene la mayor participación en el рів mundial, es el mayor exportador y sus niveles de innovación y de generación de conocimiento se acercan cada vez más a los de EUA.

Sin embargo, las instituciones chinas no han sido exportadas como líderes mundiales, la barrera del lenguaje, la particularidad de su cultura y la falta de 
conocimiento de su poderío militar hacen plantear que, si bien China presenta rasgos muy similares a los de EUA previos al fin de la Segunda Guerra Mundial, no parece ser el país que vaya a convertirse en hegemonía absoluta en el corto plazo.

Considerando el marco teórico de los siete puntos planteados: 1) extensión territorial, 2) poderío industrial, 3) poderío financiero, 4) ventaja en ciencia y tecnología, 5) poderío político-militar, 6) liderazgo cultural, y 7) incidencia internacional a partir del sistema internacional de Estados o instituciones y organizaciones internacionales y el análisis de los dos procesos de transición hegemónica se concluye lo siguiente:

- La transición de la hegemonía es un proceso histórico de largo alcance, el cual es producto de dos factores esenciales: el debilitamiento interno y externo de la potencia hegemónica, y el surgimiento de la nueva potencia que disputa el liderazgo, el cual se expresa en el fortalecimiento interno y externo de este país o conjunto de países. En el caso de la decadencia de la hegemonía inglesa, las guerras mundiales sirvieron de factor catalizador de la transición, la disputa por la hegemonía entre Reino Unido, Alemania y EUA, no obstante, EUA ya presentaba superioridad económica en muchos rubros antes de las guerras (véanse los cuadros 1 y 2). La vastedad del territorio estadounidense, a diferencia de Alemania, fue un factor determinante en la consolidación de la nueva hegemonía.

- El proceso de transición, de acuerdo con Arrighi y Silver (2001), es de por lo menos 30 años y está compuesto por tres etapas: "crisis de hegemonía, colapso de la hegemonía y nueva hegemonía". De la misma forma, autores como Hobsbawm (2004) y Dabat (2017) plantean una periodización basada en profundas transformaciones en el ámbito económico, político, social, tecnológico, geopolítico y en términos de hegemonía y gobernanza mundial. La etapa informática-global comenzó en 1980 y se encuentra en un franco periodo de declinación, lo que implica una transformación profunda que probablemente genere el fin de la hegemonía estadounidense y transite hacia un mundo multipolar (Dabat en prensa).

- En ambos procesos de transición se observa que el avance del país que se incorpora al liderazgo mundial se manifiesta de manera temprana en la manufactura, en la productividad, en la formación bruta de capital fijo, en la participación en las exportaciones mundiales y en el peso en el PIB mundial, al igual que EUA lideraba al mundo desde inicios del siglo xx, China lo hace desde inicios del siglo actual, lo cual parece ser una tendencia irrefrenable. 
- En el aspecto monetario y financiero, el cual se basa más en elementos de carácter cualitativo como la confianza, se retrasa el liderazgo de la nueva potencia emergente. La potencia en declive sustenta su poderío en una proporción importante en este sector, al igual que en el aspecto militar, se observa claramente una similitud entre la transición inglesa-estadounidense, como en la actual crisis de hegemonía estadounidense, hoy el dólar en su función de dinero mundial y el poder militar global son los dos grandes ejes que mantienen la hegemonía de EUA (Leal, 2018).

- Asimismo, se observa que ante el declive de Inglaterra como potencia líder existió una burbuja financiera que buscaba reanimar el liderazgo de la economía hegemónica (Arrighi y Silver, 2001), y en el caso de la crisis de hegemonía actual, las burbujas punto-com, hipotecaria y la everything bubble actual, han ocurrido en el momento en que China rebasó a EUA en el aspecto productivo.

- Las instituciones que gobiernan la economía mundial responden a los intereses de la nación hegemónica prevaleciente, y no responden a escenarios futuros; en la transición aún se expresa ese liderazgo, sin que se observe claramente el declive de dicha potencia. Por ejemplo, el patrón oro se mantuvo por intereses ingleses, mientras que organismos internacionales como la ONU, la OMC, el FMI, la OTAN, entre otros, siguen respondiendo en su mayoría a los intereses de EUA aún en esta crisis de hegemonía.

- A diferencia de las otras transiciones de hegemonía, en la actual no existe una guerra de carácter mundial que catalice la transición, por lo que la caída definitiva de EUA, o el surgimiento de un orden bipolar o multipolar, podría tardar varios años más en consolidarse.

- Toda lucha hegemónica presupone lucha por la expansión territorial. Mientras EUA se expandió con México, Hawái, Alaska y Puerto Rico; en el caso de China la expansión se ha realizado mediante la IED y la exportación de sus bienes, pero también es una potencia regional que ha recuperado los territorios de Hong Kong y Taiwán. Por ello, se concluye que una nación hegemónica siempre ha sido una nación con un amplio territorio, por lo que en las condiciones territoriales actuales de países avanzados como Corea, Japón o Alemania, se observan escasas posibilidades de fungir como hegemonía.

- EUA ha pretendido mantener su hegemonía basándose en la intimidación militar y económica, por lo que un cambio hacia un mundo multipolar o bipolar es un avance en términos de democracia e igualdad de derechos entre las naciones. 
- Existe una posibilidad de que EUA realice actos de provocación contra China para generar una Tercera Guerra Mundial que fortalezca su hegemonía (Arrighi, 2007).

- En las condiciones actuales no se observa una posibilidad real de que China tome el lugar de EUA como hegemonía mundial. En términos de importantes estudiosos de la hegemonía, no basta con tener liderazgo económico y comercial, se requiere además de liderazgo monetario, militar, cultural e institucional. En este sentido, el idioma, las instituciones, la cultura y la forma de gobierno chino son muy particulares y se observa difícil que de manera mayoritaria los países las busquen adoptar. A diferencia, la hegemonía inglesa o estadounidense sí dominaron en estos rubros, por lo que se reitera que se está ante una profunda crisis de la hegemonía estadounidense de la cual difícilmente se pueda recuperar, pero pretender que se acerca a una hegemonía china no es viable. Sin embargo, se plantea la posibilidad de un mundo multipolar en el sentido de que avancen junto a China países como Rusia y Alemania, acompañando el liderazgo estadounidense en un mundo menos vertical.

\section{BIBLIOGRAFÍA}

Agencia Central de Inteligencia (CIA) (2019), The Wold Fact-Book. Consultado [26 de julio de 2019]. Disponible en <https://www.cia.gov/library/publications/the-world-factbook/>

Arias, J. (2019), Bancos más grandes del mundo 2018, en "Economipedia", consultado [22 de junio de 2018]. Disponible en <https://www.universidaddebolsa.com/blog/bolsas-de-valores-mas-grandes-del-mundo>

Arrighi, G. (1999), El largo siglo XX. Dinero y poder en los origenes de nuestra época, España, Ediciones Akal.

(2007), Adam Smith en Pekin, moderno, España, Ediciones Akal.

Arrighi, G. y Silver, B. J. (2001), Caos y orden en el sistema-mundo moderno, España, Ediciones Akal.

Banco Mundial (2019), DataBank, Grupo Banco Mundial, Estados Unidos, consultado [26 de julio de 2019]. Disponible <https://datos.bancomundial.org/>

(2018), Subcriptions and voting powers of member countries, Estados Unidos de América, Secretaria Coorporativa Grupo Banco Mundial.

Bankinter (29 de septiembre de 2016), "La industria del videojuego a nivel mundial: ventas de videojuegos, Redacción”, consultado [06 de junio 
de 2019]. Diponible en <https://blog.bankinter.com/economia/-/noticia/2016/9/29/videojuegos-2016-datos>

Bairoch, P. (1982), "International industrialization levels from 1750 to 1980", Journal of European Economic History, vol. 11, núm. 2, Italia, Banco di Roma.

Braudel, F. (1984), Civilización material, economía y capitalismo, siglos XVXVIII, España, Alianza Editorial.

Brinkley, A. (2008) [1961], American history: a survey, EUA, McGraw Hill Book.

Conferecia de las Naciones Unidas sobre Comercio y Desarrollo (UNCTAD) (2019), "Informe sobre las Inversiones en el Mundo 2018", Naciones Unidas, Estados Unidos-Suiza.

Dabat, A. (2017), "Esbozo de método para el estudio del siglo xx e inicios del xxI”, Economía Informa, vol. 407, núm. C., México, UnAM, noviembrediciembre.

(en prensa), Del agotamiento del neoliberalismo a un mundo multipolar e incluyente, México, UnAM.

Dabat, A. y Leal, P. (2013), "Declinación de EU: contexto histórico mundial", Problemas del Desarrollo. Revista Latinoamericana de Economia, vol. 44, núm. 174, México, unam, julio-septiembre.

y Leal, P. (en prensa), "La declinación histórica de Estados Unidos y de su hegemonía", Del agotamiento del neoliberalismo a un mundo multipolar e incluyente, México, UNAM.

, Leal, P. y Romo, S. (2012), "Crisis mundial, agotamiento del neoliberalismo y de la hegemonía norteamericana: contexto internacional y consecuencias para México", Norteamérica, vol. 7, núm. 2, México, CISANUNAM, julio-diciembre.

Expansión (2019), "Deuda Pública", DatosMacro.com. Disponible en <https:// datosmacro.expansion.com/deuda>

Eberhard, D., Simons, G. y Fennig, C. (2019), Ethnologue: languages of the world, 22a edición, EUA, IL International.

Fondo Monetario Internacional (FMI) (2019), "IMF Members' Quotas and Voting Power, and IMF Board of Governors", Junta de Gobernadores, 05 de junio de 2019, Estados Unidos.

Frieden, J. (2007), Capitalismo global: el trasfondo económico de la historia del siglo XX, España, Memoria Crítica.

Gilpin, R. (1987), La economía politica de las relaciones internacionales, Argentina, Grupo Editor Latinoamericano. 
Global Fire Power (2019), "Grp Countries Index”. Consultado [06 de junio de 2019]. Disponible en <https://www.globalfirepower.com/countries.as> Global Trends (2012), Global Trends 2030: Alternative worlds, EUA, National Intelligence Council.

Hobsbawm, E. (2004), Historia del siglo XX: 1914-1991, España, Ediciones Crítica.

(2013), Trilogía de Hobsbawm: La era de la revolución, La era del capital, La era del imperio, Argentina, Ediciones Crítica.

Kennedy, P. (1995) [1987], Auge y caída de las grandes potencias, España, Plaza $\&$ Janes.

(1998), Hacia el siglo XXI, España, Plaza \& Janes.

Keohane, R. (1984), After hegemony. Cooperation and discord in the world political economy, EUA, Princeton University Press.

Landes, D. (1979), Progreso Tecnológico y Revolución Industrial, España, Editorial Tecnos.

Leal, P. (2018), "Notas sobre la transformación de la crisis económica en crisis política en Estados Unidos y consecuencias para México", Economía Informa, núm. 412, México, Facultad de Economía, unAM, septiembreoctubre.

Maddison, A. (1986), Las fases del desarrollo capitalista: historia económica cuantitativa, México, Colmex/FCE.

(2002), "The nature of US economic leadership: a historical and comparative view", Two hegemonies: Britain 1846-1914 and the United States 1941-2001, Inglaterra, Routledge.

Marichal, C. (2010), Nueva historia de las grandes crisis financieras, 18732008, México, Editorial Debate.

Nye, J. S. Jr. (2003), La paradoja del poder norteamericano, Chile, Taurus.

Organización de las Naciones Unidas (ONU) (2019), "Miembros Actuales, Consejo de Seguridad de la onu", consultado [06 diciembre de 2018]. Disponible en <https://www.un.org/securitycouncil/es/content/currentmembers>

(1960), The International Trade Statistics Yearbook, EUA, ONU.

Organización de las Naciones Unidas para la Educación, la Ciencia y la Cultura (UNESCO) (2019), "Percentage of GBO of all films feature exhibited that are national", EUA, Organización de las Naciones Unidas.

Organización Mundial del Comercio (омc) (2019), Statistics Database Time Series, consultado [26 de julio de 2016]. Disponible en <http://stat.wto. org/StatisticalProgram/WSDBStatProgramHome.aspx?Language=E> 
Pinder, J. (1976), “Europe in the world economy 1920-1970”, en C. Cipolla (ed.), The fontana economic history of Europe, Inglaterra, Collins/Fontana books.

Shangai Ranking Consultancy (2015), "Academic ranking of world universities, estadística”, consultado [05 de junio de 2019]. Disponible en <http:// www.shanghairanking.com/es/ARWU2015.html>

Statista (2019a), "Ranking de los países que registraron el mayor volumen de ingresos cinematográficos del mundo en 2017 (en millones de dólares)”, consultado [06 de junio de 2019]. Disponible en <https://es.statista.com/ estadisticas/601039/volumen-de-ingresos-a-nivel-mundial-de-la-industria-del-cine/>

(2019b), "Ranking de los principales mercados de la música a nivel mundial según los ingresos generados en 2014 (en millones de dólares)", consultado [06 de junio de 2019]. Disponible en <https://es.statista.com/ estadisticas/600755/principales-mercados-de-la-musica-a-nivel-mundial-por-ingresos/>

TradeMap (2019), "Estadísticas del comercio para el desarrollo internacional de las empresas”, EUA, International Trade Centre.

Voz Libre (06 de septiembre de 2018), "Los 10 países del mundo con más embajadas en el extranjero", en Voz Libre Portal de Internet, Sección Redacción, consultado [06 de diciembre de 2018]. Disponible en <https:// vozlibre.com/embajadas/los-10-paises-del-mundo-mas-embajadas-extranjero-18586/>

Wallerstein, I. (2007), Geopolitica y geocultura: ensayos sobre el moderno sistema mundial, España, Kairos.

Weber, M. (2003), La ética protestante y el espiritu del capitalismo, México, Fondo de Cultura Económica.

YouBioIt.com (2019), "Cantidad de premios Nobel por país", consultado [05 de junio de 2019]. Disponible en <https://www.youbioit.com/es/article/ shared-information/21057/cantidad-de-premios-nobel-por-pais> 